Supporting Information for

\title{
Assessing the Effects of Temperature and Oxygen Vacancy on Band Gap Renormalization in $\mathrm{LaCrO}_{3-\delta}$ : First-Principles and Experimental Corroboration
}

Jongwoo Park ${ }^{1}$, Wissam A. Saidi ${ }^{1,2}$, Jeffrey K. Wuenschell1 ${ }^{1,3}$, Bret H. Howard ${ }^{1}$, Benjamin

Chorpening ${ }^{1}$, and Yuhua Duan ${ }^{1 *}$

${ }^{1}$ National Energy Technology Laboratory, United States Department of Energy, Pittsburgh, PA 15236 USA

2 Department of Mechanical Engineering and Materials Science, University of Pittsburgh, Pittsburgh, PA 15261 USA

${ }^{3}$ Leidos Research Support Team, Pittsburgh, PA 15236 USA

*Corresponding author. E-mail: yuhua.duan@netl.doe.gov.

\section{Table of Contents}

S1. Simulation Details

Figure S1. Structural configurations of orthorhombic Pnma, rhombohedral $R 3 c$, and cubic Pm $3 m \mathrm{LaCrO}_{3}$. Polyhedral planes are shown around each $\mathrm{La}$ and $\mathrm{Cr}$ cation. Structures are depicted as a function of temperature with the phase transition temperatures notated. Relative ground-state energy from different magnetic orderings is summarized as compared to AFM-G ordering marked in bold (data reproduced from Figure 1a of the main text for cubic $\mathrm{LaCrO}_{3}$ ).

Table S1. Optimized lattice parameters, magnetic moments, and band gaps for orthorhombic, rhombohedral, and cubic $\mathrm{LaCrO}_{3}\left(o-\mathrm{LaCrO}_{3}, r-\mathrm{LaCrO}_{3}\right.$, and $c$ - $\left.\mathrm{LaCrO}_{3}\right)$ calculated by VASP and ABINIT.

S2. Electronic Band Structures of Oxygen-Vacant $c-\mathrm{LaCrO}_{3-\delta} \mathrm{v}_{\mathrm{O}}{ }^{q}$

Figure S2. Electronic band structures along the high-symmetry paths in the first Brillouin zones and PDOS for oxygen-vacant cubic $\mathrm{LaCrO}_{3-\delta}\left(c-\mathrm{LaCrO}_{3}\right) \mathrm{v}_{0} q$. Zero of the energy $(E)$ scale is position of the Fermi level $\left(E_{\mathrm{F}}\right)$ set in the middle between the band edges.

S3. Phonon and Zero-Point-Motion Renormalization Calculations

Figure S3. Helmholtz free energy, entropy and constant-volume heat capacity of pristine (a) orthorhombic $\mathrm{LaCrO}_{3}\left(o-\mathrm{LaCrO}_{3}\right)$ and (b) rhombohedral $\mathrm{LaCrO}_{3}\left(r-\mathrm{LaCrO}_{3}\right)$.

Figure S4. Band gap renormalization $\left(\Delta E_{\mathrm{g}}\right)$ as a function of temperature on direct band gap at Gamma for pristine (a) orthorhombic $\mathrm{LaCrO}_{3}\left(o-\mathrm{LaCrO}_{3}\right)$ and (b) rhombohedral $\mathrm{LaCrO}_{3}$ 
$\left(r-\mathrm{LaCrO}_{3}\right)$ shown with convergence with respect to $q$-point grids.

Figure S5. (a) Phonon frequency dispersions along the high-symmetry line and phonon PDOS for oxygen-vacant cubic $\mathrm{LaCrO}_{3-\delta}\left(c-\mathrm{LaCrO}_{3}\right) \mathrm{v}_{O} q$. (b) Helmholtz free energy, entropy, and constant-volume heat capacity of oxygen-vacant $c-\mathrm{LaCrO}_{3} \mathrm{~V}_{\mathrm{O}} q$ as a function of temperature.

Figure S6. (a) Convergence on VBM, CBM, and band gap renormalization $\left(\Delta E_{\mathrm{g}}\right)$ with respect to $q$-point grids for pristine cubic $\mathrm{LaCrO}_{3}\left(c-\mathrm{LaCrO}_{3}\right)$. (b) Convergence on $\Delta E_{\mathrm{g}}$ with respect to $q$-point grids for oxygen-vacant $c-\mathrm{LaCrO}_{3} \mathrm{v}_{\mathrm{O}}$.

Figure S7. Finite-size effect on the temperature band gap renormalization $\left(\Delta E_{\mathrm{g}}\right)$ on direct band gap at Gamma for oxygen-vacant cubic $\mathrm{LaCrO}_{3-\delta}\left(c-\mathrm{LaCrO}_{3}\right) \mathrm{v}_{0} q$.

S4. Temperature Dependence of the Optical Band Gap Measurements in $\mathrm{LaCrO}_{3}$

Figure S8. (a) High-temperature XRD spectra of $\mathrm{LaCrO}_{3}$ sample (left: spectra from 20 to 35 degrees 2 $\theta$, right: zoomed-in spectra at around 32.5 degrees 2 $\theta$ ). (b) Direct-gap Tauc plots for pristine $\mathrm{LaCrO}_{3}$ sample, calculated from the transmission measurements at elevated temperatures. The linear regions of each curve are extrapolated (shown as dotted lines) determining the optical absorption edges.

S5. Applicability of Analytical Modeling on the Band Gap Variance

Figure S9. Temperature dependence of $E_{\mathrm{g}}$ for pristine and oxygen-vacant $\left(\mathrm{v}_{\mathrm{O}}{ }^{q}\right)$ cubic $\mathrm{LaCrO}_{3-\delta}\left(c-\mathrm{LaCrO}_{3}\right)$. Symbols refer to simulated $E_{\mathrm{g}}$ and lines represent the Varshni fits.

Table S2. $E_{\mathrm{o}}, \alpha$, and $\beta$ in the Varshni equation for fitting temperature dependence of the band gaps via the $\mathrm{AHC}$ simulations in pristine and oxygen-vacant $\left(\mathrm{v}_{\mathrm{O}}^{q}\right)$ cubic $\mathrm{LaCrO}_{3-\delta}(c$ $\mathrm{LaCrO}_{3}$ ). Fitness of the Varshni equation is measured with $R^{2}$.

Figure S10. Temperature dependence of simulated and experimental $E_{\mathrm{g}}$ for pristine $\mathrm{LaCrO}_{3}$ (reproduced from Figure 4). Red lines on top of experiment data show the O'Donnell fits: dashed for orthorhombic $\mathrm{LaCrO}_{3}\left(o-\mathrm{LaCrO}_{3}\right)$ and solid for rhombohedral $\mathrm{LaCrO}_{3}(r-$ $\mathrm{LaCrO}_{3}$ ), as a reference to a phase transition temperature.

Table S3. $E_{0}, S$, and $<\hbar \omega>$ in the O'Donnell equation for fitting temperature dependence of the band gaps via the in-house experiment in pristine $\mathrm{LaCrO}_{3}$. Measured band gaps are fitted for orthorhombic and rhombohedral $\mathrm{LaCrO}_{3}\left(o-\mathrm{LaCrO}_{3}\right.$ and $\left.r-\mathrm{LaCrO}_{3}\right)$ with respect to a phase transition temperature. Fitness of the $\mathrm{O}^{\prime}$ Donnell equation is measured with $R^{2}$.

\section{References}




\section{S1. Simulation Details}

$\mu_{\mathrm{O}}(T, P)$ in the formation energy for oxygen mono-vacancy (Eq. (1) in the main text) is calculated using Eq. (S1). We employ the ideal gas assumption that the surrounding oxygen atmosphere forms an ideal-gas like reservoir. The chemical potential of an oxygen $\mu_{\mathrm{O}}(T, P)$ in the bulk perovkites is identical to that in the gas phase at equilibrium conditions. ${ }^{1,2}$

$$
\mu_{O}(T, P)=\frac{1}{2} \mu_{O_{2}}(T, P)=\mu_{O}\left(T, P_{0}\right)+\frac{1}{2} k_{B} T \ln \frac{P}{P_{0}}
$$

$k_{\mathrm{B}}$ is the Boltzmann constant. To calculate the reference state of $\mu_{\mathrm{O}}(T, P)$ at $T=0 \mathrm{~K}$ and $P=P_{0}$, the total energy of an isolated oxygen $\left(E_{\mathrm{O} 2}\right)$ is used that is calculated at PBE-GGA level, ${ }^{3}$ where $\mu_{\mathrm{O}}\left(0, P_{0}\right)=1 / 2 \mu_{\mathrm{O} 2}\left(0, P_{0}\right)=1 / 2 E_{\mathrm{O} 2}$. Considering the correction of $\Delta h$ for PBE-GGA calculated $E_{\mathrm{O} 2}, \Delta h=1.36 \mathrm{eV} / \mathrm{O}_{2},{ }^{3}$ the resulting $\mu_{\mathrm{O}}(T, P)$ becomes $\mu_{\mathrm{O}}\left(0, P_{0}\right)=1 / 2\left(E_{\mathrm{O} 2}+\Delta h\right)=-4.25 \mathrm{eV} / \mathrm{O}$. $\mu_{\mathrm{O}}\left(T, P_{0}\right)$ at $T=1300 \mathrm{~K}$ (i.e. the phase transition temperature into a cubic $\left.\mathrm{LaCrO}_{3}{ }^{4}\right)$ was derived from $\mu_{\mathrm{O}}\left(T, P_{0}\right)=\mu_{\mathrm{O}}\left(0, P_{0}\right)+1 / 2 \Delta G_{\mathrm{O} 2}\left(T, P_{0}\right) . \Delta G_{\mathrm{O} 2}\left(T, P_{0}\right)$ is determined from the thermodynamic data of the NIST-JANAF thermochemical tables, ${ }^{5}$ resulting in $\mu_{\mathrm{O}}\left(1300, P_{0}\right)=-5.66 \mathrm{eV}$. The vibrational free energy of an oxygen atom in the gas phase is taken account in $\mu_{\mathrm{O}}(T, P)$. A correction term $E_{\text {corr }}$ is calculated as implemented in the python charged defect toolkit (PyCDT) ${ }^{6}$ $\Delta F^{q}(T)$ is the phonon free energy difference between defective and pristine structures, $\Delta F^{q}(T)=$ $F^{q}\left(\mathrm{ABO}_{3}, \mathrm{v}_{0} q, T\right)-F\left(\mathrm{ABO}_{3}, T\right)$.

For $\mathrm{DFT}+U$, phonon, and $\mathrm{AHC}$ simulations of pristine orthorhombic and rhombohedral $\mathrm{LaCrO}_{3}, 20$-atom and 30-atom primitive cells with $7 \times 4 \times 7$ and $7 \times 7 \times 1$ Monkhorst-Pack $k$ point mesh grids are used for each structure, respectively. The rest of computational criteria are held in line with those of cubic $\mathrm{LaCrO}_{3}$. Figure $\mathrm{S} 1$ shows the schematic illustration of orthorhombic-rhombohedral-cubic phase transition of $\mathrm{LaCrO}_{3},{ }^{4}$ and magnetic structure calculation results in each phase. In all phases, $\mathrm{LaCrO}_{3}$ is favored in AFM-G ordering and show band gaps over $2 \mathrm{eV}$ (see Table S1) that are in fair agreements with previous studies., ${ }^{2,7,8}$

Table S1 summarizes optimized lattice parameters, local magnetic moments, and direct band gap energies calculated by VASP and ABINIT for AFM-G ordered orthorhombic, rhombohedral, and cubic $\mathrm{LaCrO}_{3}$. The quantitative agreements on these ground-state properties between VASP and ABINIT, in part, suggest the reliable AHC calculation via ABINIT that uses the normconserving pseudopotentials. As a side note, the lattice parameters remain identical for an oxygen-vacant cubic $\mathrm{LaCrO}_{3-\delta}$ structure, due to marginal effect of removing an atom on structural distortion from a supercell we modeled. 


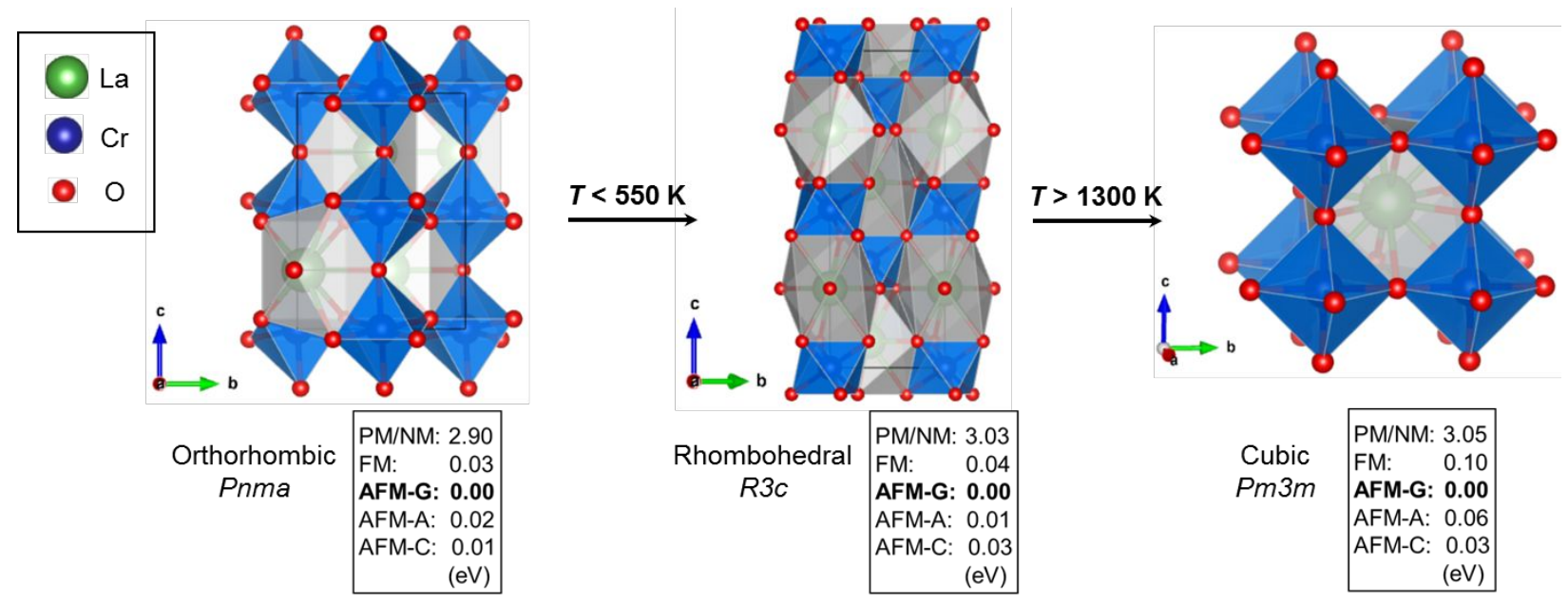

Figure S1. Structural configurations of orthorhombic Pnma, rhombohedral $R 3 c$, and cubic $P m 3 m \mathrm{LaCrO}_{3}$. Polyhedral planes are shown around each $\mathrm{La}$ and $\mathrm{Cr}$ cation. Structures are depicted as a function of temperature with the phase transition temperatures notated. Relative ground-state energy from different magnetic orderings is summarized as compared to AFM-G ordering marked in bold (data reproduced from Figure 1a of the main text for cubic $\mathrm{LaCrO}_{3}$ ).

Table S1. Optimized lattice parameters, magnetic moments, and band gaps for orthorhombic, rhombohedral, and cubic $\mathrm{LaCrO}_{3}\left(o-\mathrm{LaCrO}_{3}, r-\mathrm{LaCrO}_{3}\right.$, and $\left.c-\mathrm{LaCrO}_{3}\right)$ calculated by VASP and ABINIT.

\begin{tabular}{|c|c|c|c|c|c|c|}
\hline & & \multicolumn{3}{|c|}{ Lattice Parameter $(\AA)$} & \multirow{2}{*}{$\begin{array}{l}\text { Magnetic Moment } \\
\left(\mu_{\mathrm{B}} / \text { atom }\right)\end{array}$} & \multirow{2}{*}{$\begin{array}{l}\text { Band Gap } \\
(\mathrm{eV})\end{array}$} \\
\hline & & $a$ & $b$ & $c$ & & \\
\hline \multirow{2}{*}{$o-\mathrm{LaCrO}_{3}$} & VASP & 5.598 & 5.561 & 7.877 & 2.87 & $2.88^{\mathrm{a}}$ \\
\hline & ABINIT & 5.602 & 5.564 & 7.886 & 2.82 & 2.71 \\
\hline \multirow{2}{*}{$r-\mathrm{LaCrO}_{3}$} & VASP & 5.611 & 5.611 & 13.413 & 2.73 & $2.96^{\mathrm{b}}$ \\
\hline & ABINIT & 5.618 & 5.618 & 13.433 & 2.64 & 2.84 \\
\hline \multirow{2}{*}{$c-\mathrm{LaCrO}_{3}$} & VASP & 7.838 & 7.838 & 7.838 & 3.00 & $2.62^{\mathrm{c}}$ \\
\hline & ABINIT & 7.854 & 7.854 & 7.854 & 2.97 & 2.55 \\
\hline
\end{tabular}

a,b,c $E_{\mathrm{o}}$ used to calculate the simulated band gaps $E_{\mathrm{g}}$ at given temperature on account of the zeropoint and finite temperature band gap renormalization. 


\section{S2. Electronic Band Structures of Oxygen-Vacant $c-\mathrm{LaCrO}_{3-\delta} \mathbf{v}_{0}^{q}$}

Figure S2 shows the electronic band structures, and atom- and angular-momentum projected PDOS for oxygen-vacant cubic $\mathrm{LaCrO}_{3-\delta}$ with different vacancy charges $\mathrm{v}_{0}{ }^{q}$, discussed in Figure $2 \mathrm{~b}$ of the main text. PDOS of oxygen-vacant $\mathrm{v}_{\mathrm{O}} q$ states of cubic $\mathrm{LaCrO}_{3-\delta}$ are reproduced from Figure $2 \mathrm{~b}$ for complete comparisons.
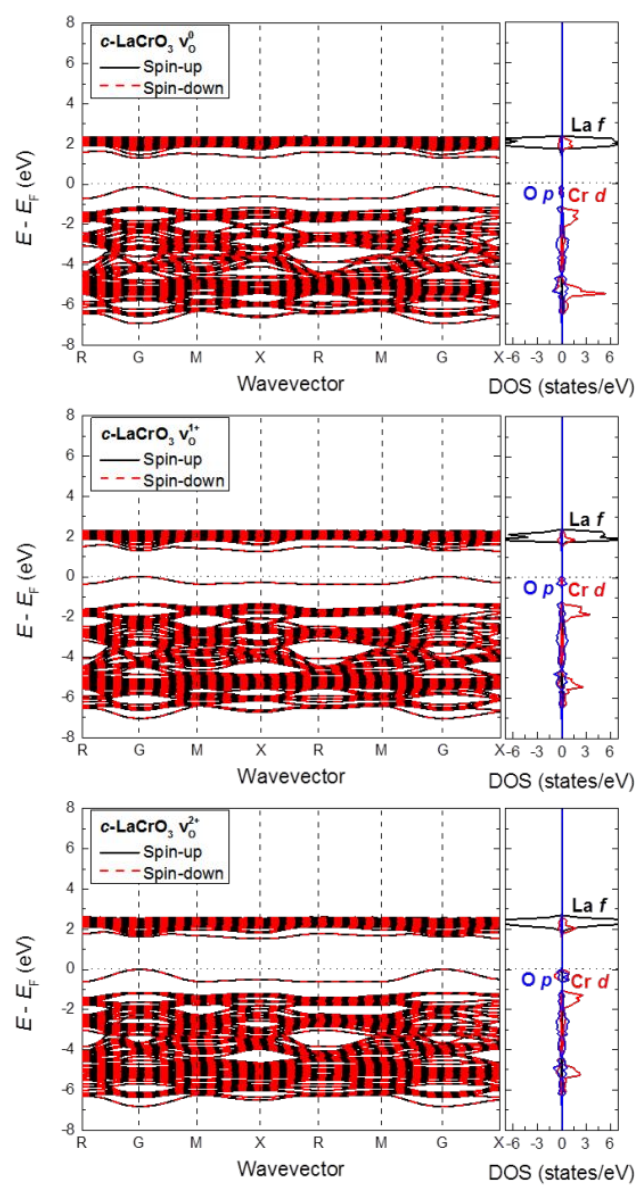

Figure S2. Electronic band structures along the high-symmetry paths in the first Brillouin zones and PDOS for oxygen-vacant cubic $\mathrm{LaCrO}_{3-\delta}\left(c-\mathrm{LaCrO}_{3}\right) \mathrm{v}_{0} q$. Zero of the energy $(E)$ scale is position of the Fermi level $\left(E_{\mathrm{F}}\right)$ set in the middle between the band edges. 


\section{S3. Phonon and Zero-Point-Motion Renormalization Calculations}

Figure S3 shows the Helmholtz free energy, entropy, and constant-volume heat capacity of phonons in orthorhombic and rhombohedral $\mathrm{LaCrO}_{3}$. For both structures, the Helmholtz free energy decreases while the entropy and heat capacity increase (from 0 at zero-temperature) as a function of temperature, all of which obey the third law of thermodynamics. Such insights also help obtaining various thermophysical properties of materials (e.g. the Debye temperature), although doing so is not the scope of this work.
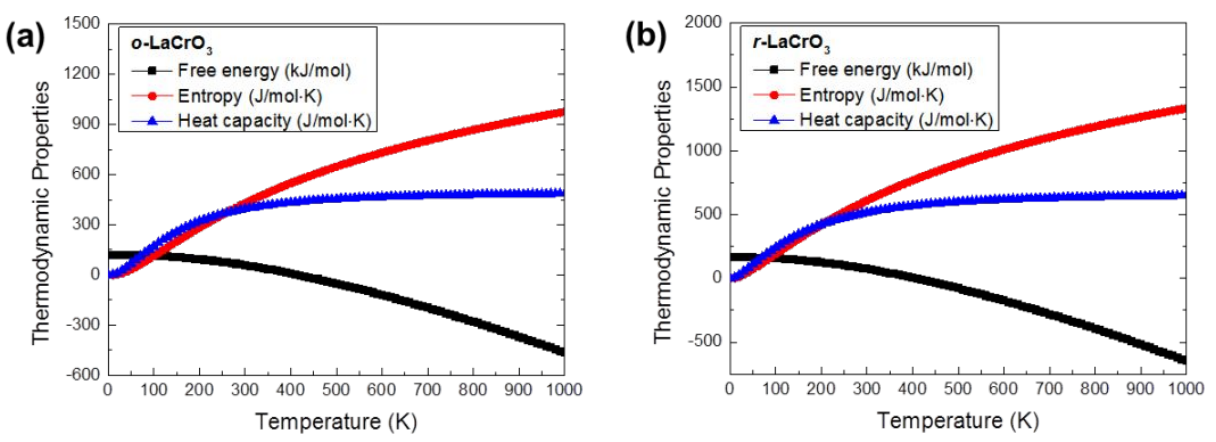

Figure S3. Helmholtz free energy, entropy and constant-volume heat capacity of pristine (a) orthorhombic $\mathrm{LaCrO}_{3}\left(o-\mathrm{LaCrO}_{3}\right)$ and (b) rhombohedral $\mathrm{LaCrO}_{3}\left(r-\mathrm{LaCrO}_{3}\right)$.

Figure S4 shows the band gap renormalization in pristine orthorhombic and rhombohedral $\mathrm{LaCrO}_{3}$ (discussed in Figure 4 of the main text), and its convergence with respect to $q$-point grid. At converged condition of $25 \times 25 \times 25 q$-point grid, the zero-point renormalization is $-0.13 \mathrm{eV}$ and $-0.27 \mathrm{eV}$ in orthorhombic and rhombohedral $\mathrm{LaCrO}_{3}$, respectively. The band gaps monotonically decrease in both phases, where the finite temperature renormalization at $1000 \mathrm{~K}$ corresponds to $-0.92 \mathrm{eV}$ in orthorhombic and $-0.93 \mathrm{eV}$ in rhombohedral phase.
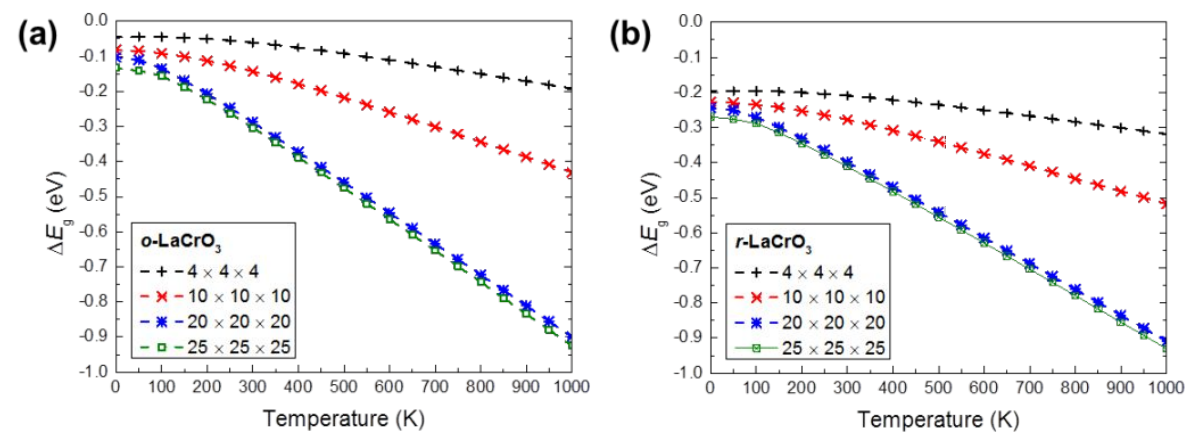

Figure S4. Band gap renormalization $\left(\Delta E_{\mathrm{g}}\right)$ as a function of temperature on direct band gap at Gamma for pristine (a) orthorhombic $\mathrm{LaCrO}_{3}\left(o-\mathrm{LaCrO}_{3}\right)$ and (b) rhombohedral $\mathrm{LaCrO}_{3}(r-$ $\mathrm{LaCrO}_{3}$ ) shown with convergence with respect to $q$-point grids. 
Figure S5 shows phonon dispersions and thermodynamic quantities in cubic $\mathrm{LaCrO}_{3-\delta} \mathrm{v}_{\mathrm{O}} q$. The potential localized phonon states near the vacancy was not considered due to its marginal effect. ${ }^{1}$
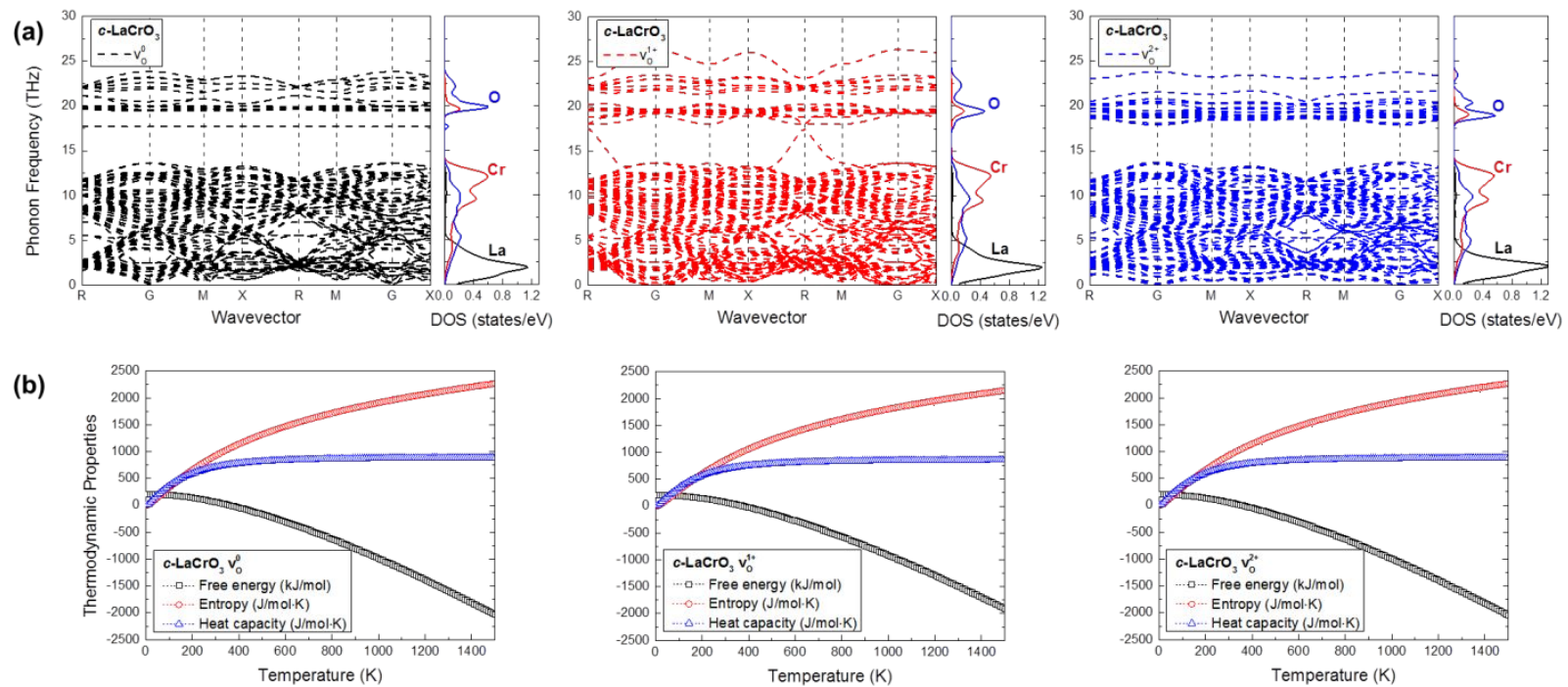

Figure S5. (a) Phonon frequency dispersions along the high-symmetry line and phonon PDOS for oxygen-vacant cubic $\mathrm{LaCrO}_{3-\delta}\left(c-\mathrm{LaCrO}_{3}\right) \mathrm{v}_{\mathrm{O}}{ }^{q}$. (b) Helmholtz free energy, entropy, and constant-volume heat capacity of oxygen-vacant $c-\mathrm{LaCrO}_{3} \mathrm{v}_{\mathrm{O}}^{q}$ as a function of temperature.

Figure S6 presents convergence on the band gap renormalization in pristine and oxygenvacant cubic $\mathrm{LaCrO}_{3-\delta} \mathrm{V}_{\mathrm{O}}^{q}$ with respect to $q$-point grid to sample the first Brillouin zone.

(a)

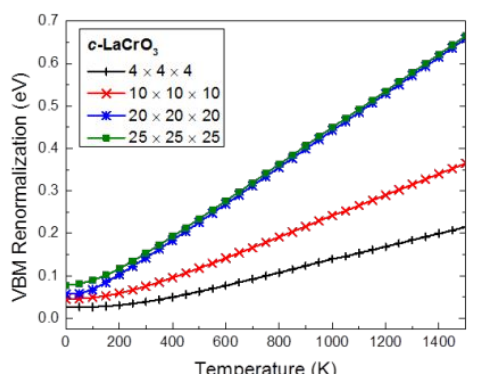

(b)

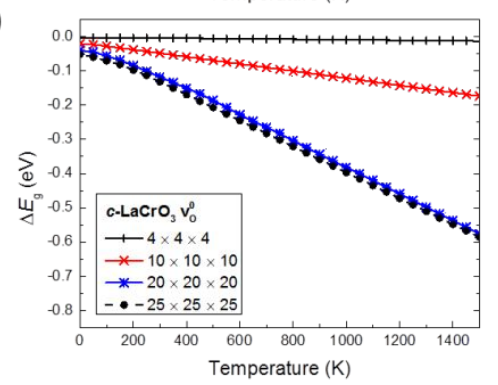

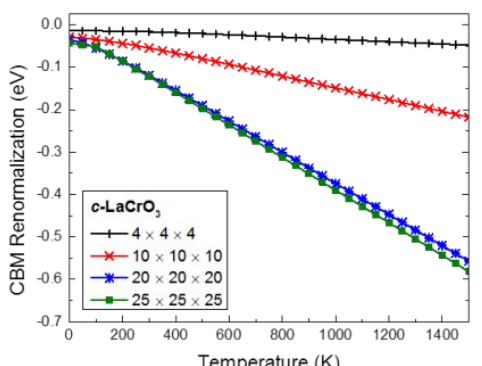
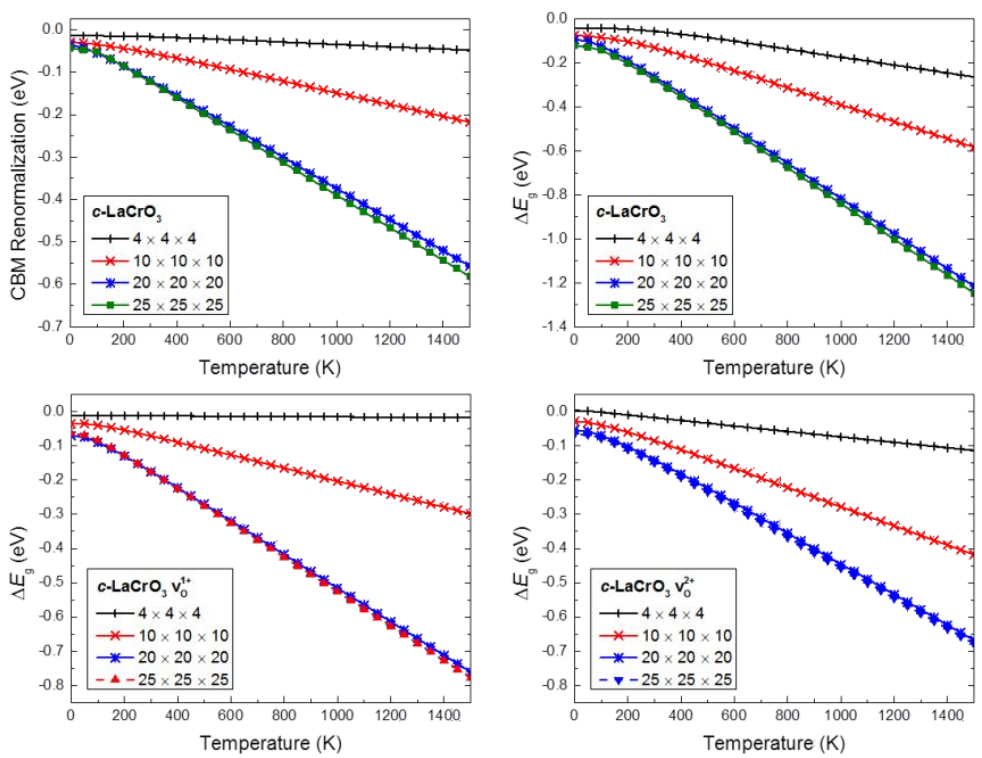

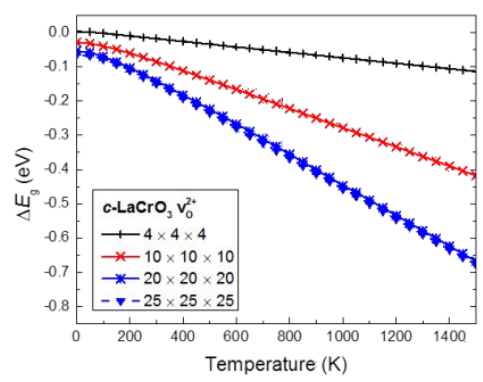

Figure S6. (a) Convergence on VBM, CBM, and band gap renormalization $\left(\Delta E_{\mathrm{g}}\right)$ with respect to $q$-point grids for pristine cubic $\mathrm{LaCrO}_{3}\left(c-\mathrm{LaCrO}_{3}\right)$. (b) Convergence on $\Delta E_{\mathrm{g}}$ with respect to $q$ point grids for oxygen-vacant $c-\mathrm{LaCrO}_{3} \mathrm{v}_{\mathrm{O}}$. 
Figure S7 illustrates finite-size effect on AHC calculations for defective cubic $\mathrm{LaCrO}_{3-\delta} \mathrm{V}_{\mathrm{O}} q$ at a $q$-point grid of $25 \times 25 \times 25$. Oxygen-vacant $\mathrm{LaCrO}_{3-\delta}$ configurations are modeled for $3 \times 3 \times 3$ and $4 \times 4 \times 4$ supercells. For $\mathrm{v}_{\mathrm{O}}{ }^{0}$, the change in renormalization is nearly negligible. $\mathrm{v}_{\mathrm{O}}{ }^{1+}$ and $\mathrm{V}_{\mathrm{O}}{ }^{2+}$ result in relatively larger variances on finite temperature corrections by $\sim 0.1 \mathrm{eV}$. The differences of finite temperature renormalization at $1500 \mathrm{~K}$ between $\mathrm{v}_{\mathrm{O}}^{q}$ decrease as the supercell size increases. However, the general trend and magnitude of band gap changes across $\mathrm{v}_{\mathrm{O}}{ }^{q}$ are not affected. $2 \times 2 \times 2$ supercell results for $\mathrm{v}_{\mathrm{O}}{ }^{q}$ are reproduced from Figure $6 \mathrm{a}$ in the main text.

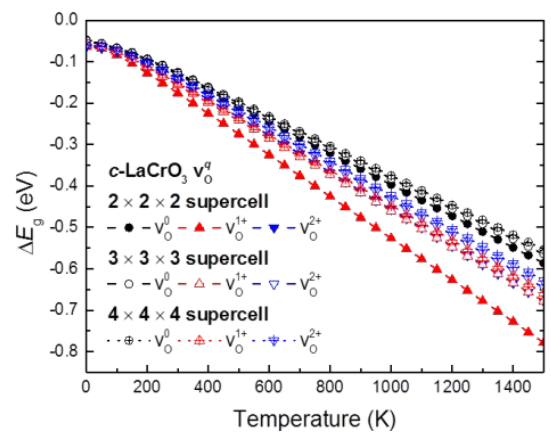

Figure S7. Finite-size effect on the temperature band gap renormalization $\left(\Delta E_{\mathrm{g}}\right)$ on direct band gap at Gamma for oxygen-vacant cubic $\mathrm{LaCrO}_{3-\delta}\left(c-\mathrm{LaCrO}_{3}\right) \mathrm{v}_{\mathrm{O}^{q}}$. 


\section{S4. Temperature Dependence of the Optical Band Gap Measurements in $\mathrm{LaCrO}_{3}$}

The crystalline phase changes of $\mathrm{LaCrO}_{3}$ are analyzed via high-temperature XRD using a PANalytical X'Pert PRO multipurpose diffractometer equipped with a $\mathrm{Cu}$ anode operated at 45 $\mathrm{kV}$ and $40 \mathrm{~mA}$, and a divergent beam monochromator. For in-situ measurement in room air, this instrument was equipped with an Anton Parr HTK1200 hot stage where the $\mathrm{LaCrO}_{3}$ powder sample was mounted in a corundum holder. Heating ramps of $20^{\circ} \mathrm{C} /$ minute were used. At each temperature, an XRD pattern was collected from 20 to 35 degrees $2 \theta$ when the set point was reached, and a second pattern was collected after a hold time of 2 hours. The following heating profile was used: analyze at ambient temperature $>$ heat to $200^{\circ} \mathrm{C}$, analyze hold analyze $>$ heat to $250^{\circ} \mathrm{C}$, analyze hold analyze $>$ heat to $300^{\circ} \mathrm{C}$, analyze hold analyze $>$ heat to $950^{\circ} \mathrm{C}$, analyze hold analyze $>$ heat to $1000^{\circ} \mathrm{C}$, analyze hold analyze $>$ heat to $1050^{\circ} \mathrm{C}$, analyze hold analyze $>$ cool to ambient temperature and analyze.

Figure S8a shows high-temperature XRD spectra of $\mathrm{LaCrO}_{3}$ powder samples prepared identically to the thin film samples used for optical characterization, i.e. using same precursor solutions, drying and annealing at $900^{\circ} \mathrm{C}$ in air (see also Section 2.3 in the main text). The diffraction peak near 32.5 degrees $2 \theta$ was used to observe phase changes. Prior works have utilized the evolution of this peak for tracking the phase transitions of $\mathrm{LaCrO}_{3}$ : orthorhombic (low-), rhombohedral (intermediate-), and cubic (high-temperature) phases. ${ }^{9}{ }^{10}$ Our results verify the as-expected phase transitions from orthorhombic to rhombohedral phase at around $250^{\circ} \mathrm{C}(\sim$ $550 \mathrm{~K})$ and from rhombohedral to cubic phase at around $950^{\circ} \mathrm{C}(\sim 1300 \mathrm{~K})$. These also demonstrate the purity and stability of $\mathrm{LaCrO}_{3}$ sample with respect to each temperaturedependent phase. As a side note, XRD spectra were taken immediately after reaching each temperature and again after a 2-hour hold time that resulted in identical spectra. This indicates that the phase transition is fast relative to the time scales of interest for sensing applications.

The oxygen stoichiometry of $\mathrm{LaCrO}_{3}$ has been heavily experimentally studied in the context of solid oxide fuel cells. It has been demonstrated to be most likely oxidized when prepared at high temperature under oxidizing conditions. For example, an in-depth study of a variety of Bsite doped $\mathrm{LaCrO}_{3}$ samples by Oishi et al. ${ }^{11}$ found that virtually all compositions of doped $\mathrm{LaCrO}_{3}$ studied to be stoichiometric at high temperature and high $\mathrm{O}_{2}$ partial pressure $P_{\mathrm{O} 2}$, i.e. $T>$ $800^{\circ} \mathrm{C}$ and $\log \left(P_{\mathrm{O} 2}\right)>-5$. Our $\mathrm{LaCrO}_{3}$ thin film samples are synthesized at higher temperature and $P_{\mathrm{O} 2}$, that is at $900^{\circ} \mathrm{C}$ in air $\left(P_{\mathrm{O} 2} \sim 0.2 \mathrm{bar}\right)$, and hence are expected to be fully oxidized (i.e. in pristine state without oxygen deficiency).

Figure $\mathrm{S} 8 \mathrm{~b}$ shows the Tauc plots for pristine $\mathrm{LaCrO}_{3}$ that result in nearly linear dependence of $(\alpha \times \epsilon)^{2}$ as a function of $\epsilon$. Tauc method determines the optical band gaps (shown in Figure 4 of the main text) under assumption that the optical absorption edge is approximately equals to the 
in-/direct band gap for semiconductors. ${ }^{12,13}$ For clarity, the plots are shown at $100^{\circ} \mathrm{C}$ intervals instead of $50^{\circ} \mathrm{C}$ intervals as for actual measurements carried out. As informed from hightemperature XRD results, variances of the absorption edges (or the band gaps) below and above $T=550 \mathrm{~K}$ correspond to those in orthorhombic and rhombohedral $\mathrm{LaCrO}_{3}$, respectively.
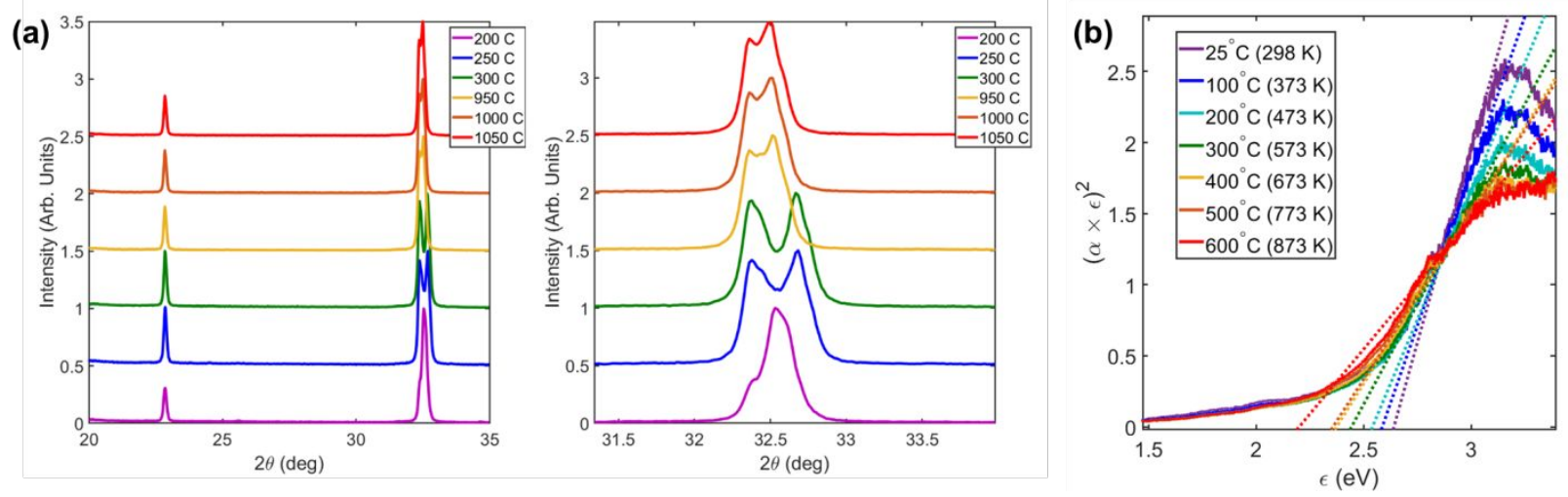

Figure S8. (a) High-temperature XRD spectra of $\mathrm{LaCrO}_{3}$ sample (left: spectra from 20 to 35 degrees 2 $\theta$, right: zoomed-in spectra at around 32.5 degrees 2 $\theta$ ). (b) Direct-gap Tauc plots for pristine $\mathrm{LaCrO}_{3}$ sample, calculated from the transmission measurements at elevated temperatures. The linear regions of each curve are extrapolated (shown as dotted lines) determining the optical absorption edges. 


\section{S5. Applicability of Analytical Modeling on the Band Gap Variance}

Varshni model is defined as Eq. (S2) ${ }^{14} E_{\mathrm{g}}$ is the in-/direct band gap at temperature $T$ and $E_{\mathrm{o}}$ is the band gap at $0 \mathrm{~K}$ (i.e. taking account of energy correction by zero-point renormalization). $\alpha$ and $\beta$ are empirical fitting parameters in the Varshni equation. Figure S9 shows the Varshni fits for simulated band gap variations in pristine and oxygen-vacant cubic $\mathrm{LaCrO}_{3-\delta}$. Zero-point renormalization corrected ground-state band gaps $E_{\mathrm{o}}$, and empirical fitting parameters $\alpha$ and $\beta$ are listed in Table S2.

$$
E_{g}=E_{o}-\alpha T^{2} /(T+\beta)
$$

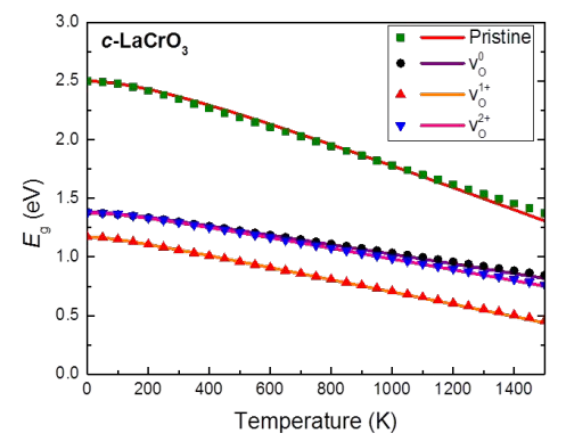

Figure S9. Temperature dependence of $E_{\mathrm{g}}$ for pristine and oxygen-vacant $\left(\mathrm{v}_{\mathrm{O}}^{q}\right)$ cubic $\mathrm{LaCrO}_{3-\delta}$ $\left(c-\mathrm{LaCrO}_{3}\right)$. Symbols refer to simulated $E_{\mathrm{g}}$ and lines represent the Varshni fits.

Table S2. $E_{0}, \alpha$, and $\beta$ in the Varshni equation for fitting temperature dependence of the band gaps via the AHC simulations in pristine and oxygen-vacant $\left(\mathrm{v}_{O} q\right)$ cubic $\mathrm{LaCrO}_{3-\delta}\left(c-\mathrm{LaCrO}_{3}\right)$. Fitness of the Varshni equation is measured with $R^{2}$.

\begin{tabular}{lllll}
\hline$c-\mathrm{LaCrO}_{3-\delta}$ & $E_{\mathrm{o}}(\mathrm{eV})$ & $\alpha\left(\times 10^{-4} \mathrm{eV} / \mathrm{K}\right)$ & $\beta(\mathrm{K})$ & $R^{2}$ \\
\hline Pristine & 2.50 & 9.9 & 370 & 0.99 \\
$\mathrm{v}_{\mathrm{O}}{ }^{0}$ & 1.38 & 4.2 & 175 & 0.99 \\
$\mathrm{v}_{\mathrm{O}}{ }^{1+}$ & 1.17 & 5.3 & 130 & 0.99 \\
$\mathrm{v}_{\mathrm{O}}{ }^{2+}$ & 1.38 & 4.7 & 190 & 0.99 \\
\hline
\end{tabular}

Considering that O'Donnell model improved the data description upon the Varshni model, especially when a given material exhibits temperature independence of the band gaps at low temperature regime instead of quadratic dependence, ${ }^{15}$ we apply O'Donnell equation to our inhouse experiment. Figure S10 presents O'Donnell fits on the temperature dependent optical band gaps in pristine $\mathrm{LaCrO}_{3}$ (data reproduced from Figure 4 of the main text). O'Donnell model efficiently applies to each orthorhombic and rhombohedral structure, considering that measured 
data below and above $T=550 \mathrm{~K}$ represents the band gaps in each phase of $\mathrm{LaCrO}_{3}$. This allows a comparison of $E_{\mathrm{o}}$ between experiment (i.e. zero-temperature band gap extracted via model fitting) and theory (i.e. zero-point renormalization corrected ground-state band gap). The experimental and computed $E_{\mathrm{o}}$ deviate less than $50 \mathrm{meV}$ in both phases of $\mathrm{LaCrO}_{3}$. Table $\mathrm{S} 3$ lists $E_{\mathrm{o}}, S$, and $<\hbar \omega>$ used for each O'Donnell fit.

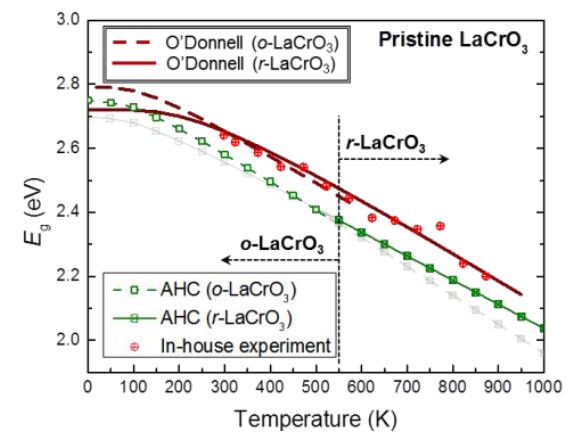

Figure S10. Temperature dependence of simulated and experimental $E_{\mathrm{g}}$ for pristine $\mathrm{LaCrO}_{3}$ (reproduced from Figure 4). Red lines on top of experiment data show the O'Donnell fits: dashed for orthorhombic $\mathrm{LaCrO}_{3}\left(o-\mathrm{LaCrO}_{3}\right)$ and solid for rhombohedral $\mathrm{LaCrO}_{3}\left(r-\mathrm{LaCrO}_{3}\right)$, as a reference to a phase transition temperature.

Table S3. $E_{0}, S$, and $<\hbar \omega>$ in the O'Donnell equation for fitting temperature dependence of the band gaps via the in-house experiment in pristine $\mathrm{LaCrO}_{3}$. Measured band gaps are fitted for orthorhombic and rhombohedral $\mathrm{LaCrO}_{3}\left(o-\mathrm{LaCrO}_{3}\right.$ and $\left.r-\mathrm{LaCrO}_{3}\right)$ with respect to a phase transition temperature. Fitness of the O'Donnell equation is measured with $R^{2}$.

\begin{tabular}{lllll}
\hline & $E_{\mathrm{o}}(\mathrm{eV})^{\mathrm{a}}$ & $S$ & $<\hbar \omega>(\mathrm{meV})$ & $R^{2 \mathrm{~b}}$ \\
\hline$o-\mathrm{LaCrO}_{3}$ & 2.79 & 5.0 & 30 & 0.91 \\
$r-\mathrm{LaCrO}_{3}$ & 2.72 & 5.2 & 60 & 0.87 \\
\hline
\end{tabular}

${ }^{\text {a }} E_{\mathrm{o}}$ are extracted to obtain an optimal $R^{2}$ of the fits while extrapolating to zero temperature.

${ }^{\mathrm{b}} R^{2}$ are calculated within each temperature regime of the measured data. 


\section{References}

(1) Jia, T.; Zeng, Z.; Zhang, X.; Ohodnicki, P.; Chorpening, B.; Hackett, G.; Lekse, J.; Duan, Y. The Influence of Oxygen Vacancy on the Electronic and Optical Properties of $\mathrm{ABO}_{3-\delta}(\mathrm{A}=$ La, Sr, B = Fe, Co) Perovskites. Phys. Chem. Chem. Phys. 2019, 21, 20454-20462.

(2) Park, J.; Wu, Y. N.; Saidi, W. A.; Chorpening, B.; Duan, Y. First-Principles Exploration of Oxygen Vacancy Impact on Electronic and Optical Properties of $\mathrm{ABO}_{3-\delta}(\mathrm{A}=\mathrm{La}, \mathrm{Sr} ; \mathrm{B}=$ Cr, Mn) Perovskites. Phys. Chem. Chem. Phys. 2020, 22, 27163-27172.

(3) Wang, L.; Maxisch, T.; Ceder, G. Oxidation Energies of Transition Metal Oxides within the GGA+U Framework. Phys. Rev. B: Condens. Matter Mater. Phys. 2006, 73, 195107.

(4) Momin, A. C.; Mirza, E. B.; Mathews, M. D. High-Temperature X-ray Diffractometric Studies of $\mathrm{LaCrO}_{3}$. J. Mater. Sci. Lett. 1991, 10, 1246-1248.

(5) Chase Jr., M. W. NIST-JANAF Thermochemical Tables. J. Phys. Chem. Ref. Data, Monogr. 1998, 9, 1-1951.

(6) Broberg, D.; Medasani, B.; Zimmermann, N. E. R.; Yu, G.; Canning, A.; Haranczyk, M.; Asta, M.; Hautier, G. PyCDT: A Python Toolkit for Modeling Point Defects in Semiconductors and Insulators. Comput. Phys. Commun. 2018, 226, 165-179.

(7) He, J.; Franchini, C. Screened Hybrid Functional Applied to $3 d^{0} \rightarrow 3 d^{8}$ Transition-Metal Perovskites $\mathrm{LaMO}_{3}(M=\mathrm{Sc}-\mathrm{Cu})$ : Influence of the Exchange Mixing Parameter on the Structural, Electronic, and Magnetic Properties. Phys. Rev. B 2012, 86, 235117.

(8) Dabaghmanesh, S.; Sarmadian, N.; Neyts, E. C.; Partoens, B. A First Principles Study of pType Defects in $\mathrm{LaCrO}_{3}$. Phys. Chem. Chem. Phys. 2017, 19, 22870-228876.

(9) Malghe, Y. S.; Dharwadkar, S. R.; Krishnan, K.; Singh Mudher, K. D. Dilatometry and High Temperature X-ray Diffractometry Study of $\mathrm{LaCrO}_{3}$ Prepared Using Microwave Heating. $J$. Therm. Anal. Calorim. 2009, 95, 49-52.

(10) Gupta, S.; Mahapatra, M. K.; Singh, P. Phase Transformation, Thermal Expansion and Electrical Conductivity of Lanthanum Chromite. Mater. Res. Bull. 2013, 48, 3262-3267.

(11) Oishi, M.; Yashiro, K.; Hong, J. O.; Nigara, Y.; Kawada, T.; Mizusaki, J. Oxygen Nonstoichiometry of B-site Doped $\mathrm{LaCrO}_{3}$. Solid State Ion. 2007, 178, 307-312.

(12) Tauc, J. Optical Properties and Electronic Structure of Amorphous Ge and Si. Mater. Res. Bull. 1968, 3, 37-46.

(13) Stenzel, O. The Physics of Thin Film Optical Spectra; Springer International: Switzerland, 2015.

(14) Varshni, Y. P. Temperature Dependence of the Energy Gap in Semiconductors. Physica 1967, 34, 149-154.

(15) O’Donnell, K. P.; Chen, X. Temperature Dependence of Semiconductor Band Gaps. Appl. Phys. Lett. 1991, 58, 2924-2926. 